http://jmscr.igmpublication.org/home/ ISSN (e)-2347-176x ISSN (p) 2455-0450 crossref DOI: https://dx.doi.org/10.18535/jmscr/v8i3.73

\title{
Comparison between Conventional Computed Tomography and High Resolution Tomography in Interstitial Lung Diseases
}

\author{
Authors \\ Dr Sowmiya.S, Dr Vijay Prabhu.R, Dr Karthika.G, Dr Yuvasree.V \\ Corresponding Author \\ Dr Vijay Prabhu.R \\ Chettinad Hospital and research Institute, Chennai, India
}

\section{Introduction}

Interstitial lung disease are a set of pulmonary disorders charecterised by inflammation and fibrosis of gas exchanging portion of the lung and diffuse abnormalities on lung radio graph. It is now generally conceded that computed tomography (CT) is superior to chest radiography in assessing the presence and extent of parenchymal abnormalities. The contribution of $\mathrm{CT}$ to the evaluation of interstitial and parenchymal lung diseases has been investigated, with special emphasis on high-resolution CT $(\mathrm{HRCT})^{(1-10)}$. Advances in CT technology now allow detailed imaging of the pulmonary parenchyma, and respective indications for conventional CT and HRCT are being defined. Performance of HRCT on state-of-the-art CT scanners requires modification of technical parameters, with special emphasis on section thickness and the reconstruction algorithm to enhance visualization of fine pulmonary parenchymal detail ${ }^{(1,7,8)}$. HRCT has been optimized for the evaluation of parenchymal diseases and may permit location of disease processes within lobules.
The aim of our study was

1) To correlate the findings of conventional computed tomography chest and high resolution computed tomography chest in interstitial diseases of lung.

2) To study the different radio graphic patterns significant in both HRCT and convention CT chest.

3) To demonstrate that high resolution computed tomography presents a true resolution in the assessment of interstitial lung disease $\mathrm{e}^{(9,10)}$.

\section{HRCT Technique}

Two technical features differentiate HRCT from conventional CT (i.e.,10 mm collimation). Firstly, in HRCT, collimation of the x-ray beam is narrower. This therefore results in a reduced section thickness and thus increases the likelihood that a very small lesion will have dimensions comparable to the size of the voxel, resulting in significantly improved spatial resolution. Secondly, unlike in conventional CT, in HRCT a dedicated algorithm is used to reconstruct the data. The 'high-frequency' algorithm takes advantage of the intrinsically high contrast environment of the lung parenchyma, so that the natural densities 
differences between aerated lungs and the interstitium are enhanced. The conspicuity of the blood vessels, small bronchi and interlobular septa is therefore greater than in conventional (thicksection) CT images. An important 'downside' of high-frequency algorithms is the increased visibility of image noise; however, in practice, this rarely hampers radiological interpretation ${ }^{(2,3,4)}$.

\section{HRCT in Diffuse Interstitial Lung Disease}

The interstitial lung disease affects mainly the lung parenchyma. Within the 'broad spectrum 'term ILD it is possible to put forth a wide range of entities.

- Firstly those which are secondary to a well-established cause.

- Secondly there remains a fascinating set of idiopathic interstitial pneumonia.

- Thirdly comes the granulomatous diseases which affects the lung parenchyma

- Fourthly a rarer group of common diffuse lung disease such as Langerhans cell histiocytosis and lymphangioleiomyomatosis. In patients with known case of ILD, HRCT improves the detection of subtle parenchymal pathologies. Further more ground-glass opacification, small cystic air spaces, traction bronchiectasis and irregular interface along bronchovascular entities and pleural surfaces are better appreciated on HRCT in comparison with $\mathrm{CT}^{(14)}$.

\section{Basic HRCT Patterns \\ Reticular Pattern}

A net-like (reticular) pattern signifies linear opacities that intersect one another. It occurs in intralobular interstitial thickening and it shows infiltration and thickening in the intralobular interstitium. Intra lobular interstitial thickening is a common HRCT finding in patients with usual interstitial pneumonia (UIP).The typical HRCT pattern in UIP are honeycombing and reticulation $^{(11)}$.

\section{Linear Pattern}

Linear pattern represents as an interlobular septum marginating as a part of secondary pulmonary lobule. Thickening of the interlobular septa is generally seen in patients with a wide range of interstitial lung diseases and results from fibrosis, interstitial fluid, and infiltration by cells. Smooth thickening is observed in patients with pulmonary edema or hemorrhage, lymphangiticcarcinomatosis, and amyloidosis. Nodular or beaded thickening is observed in sarcoidosis, lympangiticcarcinomatosis, and amyloidosis ${ }^{(6)}$.

\section{Ground-Glass Opacity}

Ground-glass opacity is denoted as 'hazy increased attenuation of the lung with preservation of bronchial and vascular margins'. It is cased due partial filling of air spaces, partial collapse of alveoli, interstitial thickening, normal expiration or increased capillary blood volume.It is a main pattern of acute interstitial pneumonia (AIP), acute or sub-acute hypersensitivity pneumonitis, pulmonary edema, pulmonary hemorrhage or drug-induced disease. Common case include UIP, nonspecific interstitial pneumonia (NSIP), desquamative interstitial pneumonia (DIP), and respiratory-bronchiolitis-associated interstitial lung disease (RB-ILD) ${ }^{(12)}$.

\section{Honeycombing}

They are nothing but cystic spaces, with thick, clearly definable fibrous walls lined by bronchiolar epithelium. They are usually $1 \mathrm{~cm}$ in diameter and their walls thickens measures between 1-3mm.It signifies the presence of terminal lung disease. Honeycombing has an atypical distribution mainly in asbestosis, sarcoidosis, NSIP, drug-related fibrosis, and hypersensitivity pneumonitis ${ }^{(13)}$.

\section{Cysts}

Cysts are commonly encountered in parenchymal diseases. They are thin-walled ( $<3 \mathrm{~mm}$ thick), well defined, air or fluid filled lesion rangingupto $1 \mathrm{~cm}$ in diameter, which has an epithelial or fibrous wall. These are generally seen in Langerhans' cell histiocytosis, 
lymphocytic interstitial pneumonia, post-infection pneumatoceles, and amyloidosis ${ }^{(7,8)}$.

\section{Nodules}

A nodular pattern appears as multiple round opacities ranging from $1 \mathrm{~mm}$ to $1 \mathrm{~cm}$ in diameter. Further characterization can be may as follows. Miliary nodules, as small as $1 \mathrm{~mm}$ in diameter, can easily be detected by HRCT, a significant advantage over plain radiography. Multiple small smooth or irregularly marginated nodules in a perilymphatic distribution are seen in sub-acute stage of extrinsic allergic alveolitis or respiratory bronchiolitis.

\section{Methodology}

The research proposal was accepted by the institutional human ethical committee of the Chettinad academy of research and education. It is a prospective study with the sample size of 30 patients with clinical diagnosis of interstitial lung disease who were referred to CT scanning in Chettinad hospitals and research institute (CHRI), Kelambakkam. The duration of case collection is from January 2018 to April 2019.

CT scan was performed with 64 detector row PHILIPS INGENUITY CORE 128 slice.Imaging parameters such as scan time, CTDI vol, SNR were considered to quantitatively compare HRCT and conventional CT chest in ILD. Qualitative assessments were made based on the radiologist views. The frequency distribution of the demographic data such as age, gender and other clinical data such as scan time, CTDI volume, interstitial lung disease detectable percentage and SNR are analyzed using MS EXCEL. The significance of HRCT is analyzed by implementing T-Test. The T-Test was used to evaluate the image quality scores of the findings. AP value of less than 0.05 was considered statistically significant.

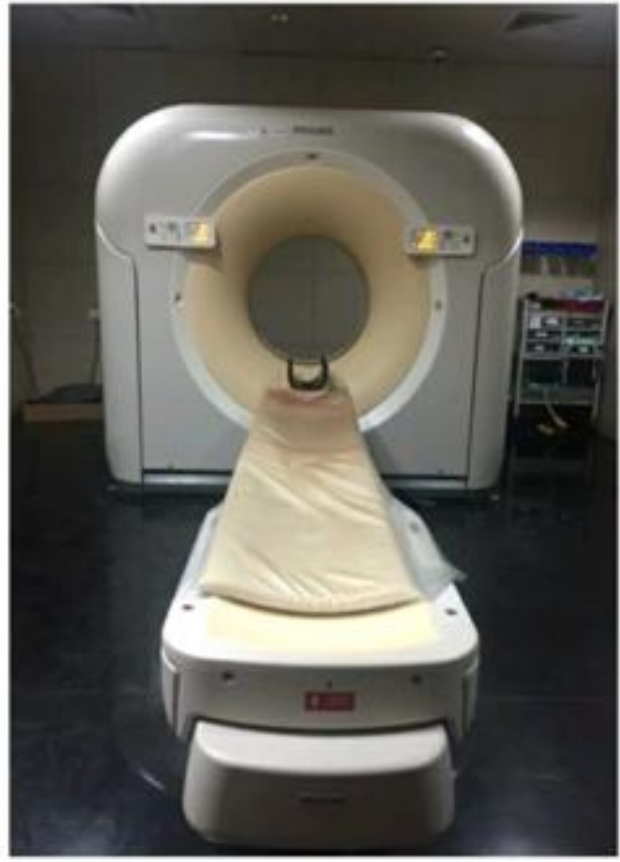

Fig 1: Philips Ingenuity 64 Detector Core 128 Slice

Table 1: Subjective Patterns of Interstitial Lung Disease

\begin{tabular}{|l|l|}
\hline PATTERN & COUNT \\
\hline Non-specific interstitial pneumonia(NSIP) & 13 \\
\hline Usual interstitial pneumonia(UIP) & 11 \\
\hline Honeycombing & 3 \\
\hline Reticular interstitial pattern & 2 \\
\hline Cryptogenic organizing pneumonia(COP) & 1 \\
\hline
\end{tabular}

\section{Results and Discussion}

Table 2: Qualitative Analysis

\begin{tabular}{|l|l|l|l|}
\hline INTERSTITIAL & CT & HRCT(VOI) & HRCT(AXIAL) \\
$\begin{array}{l}\text { LUNG DISEASE } \\
\text { DETECTED }\end{array}$ & $26 \%$ & $100 \%$ & $100 \%$ \\
PERCENTAGE & & & \\
\hline
\end{tabular}

For the past few decades, chest x-ray was the only modality to diagnose lung diseases. Gross lung parenchyma was assessed only with the advent of computed tomography. CT plays only a limited 
role in diagnosis of diffuse lung disease. Only with the advent of HRCT in 1985, different pathology and pathophysiologies of the interstitial lung diseases were delineated. The major clinical utility of HRCT is its sensitivity, specificity and accuracy in the diagnosis of interstitial disease of lung.

Table 3: Results between CT and HRCT

\begin{tabular}{|c|c|c|c|c|c|}
\hline PARAMETERS & $\begin{array}{l}\text { CT } \\
\text { (MEAN } \pm \\
\text { SD ) } \\
\mathbf{n}=\mathbf{3 0}\end{array}$ & $\begin{array}{l}\text { HRCT(Axial } \\
\text { ) } \\
(\text { MEAN } \pm \\
\text { SD) } \\
\quad n=30\end{array}$ & $\begin{array}{l}\text { HRCT } \\
\text { (Vol) } \\
\text { (MEAN } \\
\pm \text { SD) } \\
\quad \mathbf{n}=\mathbf{3 0}\end{array}$ & $\begin{array}{l}\text { P-val } \\
\text { ue } \\
\text { CT vs } \\
\text { HRC } \\
\text { T } \\
\text { (Axia } \\
\text { l) }\end{array}$ & $\begin{array}{l}\text { P-value } \\
\text { CT vs } \\
\text { HRCT } \\
\text { (Vol) }\end{array}$ \\
\hline SCANTIME & $5.7 \pm 1.76$ & $3.0 \pm 0.0$ & $6.82 \pm 1.93$ & 0.000 & 0.010 \\
\hline CTDIVOL & $10.2 \pm 1.67$ & $15.6 \pm 0.0$ & $10.35 \pm 1.92$ & 0.000 & 0.411 \\
\hline SNR & $73.96 \pm 33.15$ & $122.6 \pm 41.5$ & $98.92 \pm 32.37$ & 0.000 & 0.008 \\
\hline
\end{tabular}

In the prospective study conducted, 30 patients with clinical diagnosis of interstitial lung disease were subjected to HRCT Chest scan. CT Chest images were obtained by further reconstruction of the parent images with the respective parameters. Both the set of images were compared quantitatively based on scan time, SNR and CTDI vol and qualitatively based on the radiologist's observation.

The most common patterns observed in the study are $\mathrm{NSIP}^{(13)}$, UIP ${ }^{(11)}$, Honeycombing ${ }^{(3)}$, Reticular pattern $^{(2)}$ and $\operatorname{COP}(1)$. Based on qualitative assessment by two radiologists shows that HRCT(both axial and volume methods) results greater assessment of interstitial lung disease in comparison with conventional CT. This is principally because of 'high-frequency' algorithm that takes advantage of the intrinsically high contrast environment of the lung parenchyma and the improved spatial resolution.

Quantitative assessment based on t-test produced a statistically significant result $(\mathrm{p}=0.000)$ based on comparison of parameters such as scan time, dose levels and SNR between CT and HRCT (Axial). Dose levels of HRCT (Vol) method remains statistically insignificant $(\mathrm{p}=0.411)$. Though parameters such as scan time and SNR for HRCT (VOL) method is statistically significant $(\mathrm{p}=0.010$ and $\mathrm{p}=0.000$ ). A potential limitation to this study is the dose levels.

In summary, the current study proves that HRCT protocol is the gold standard method in diagnosis of interstitial diseases of lung.

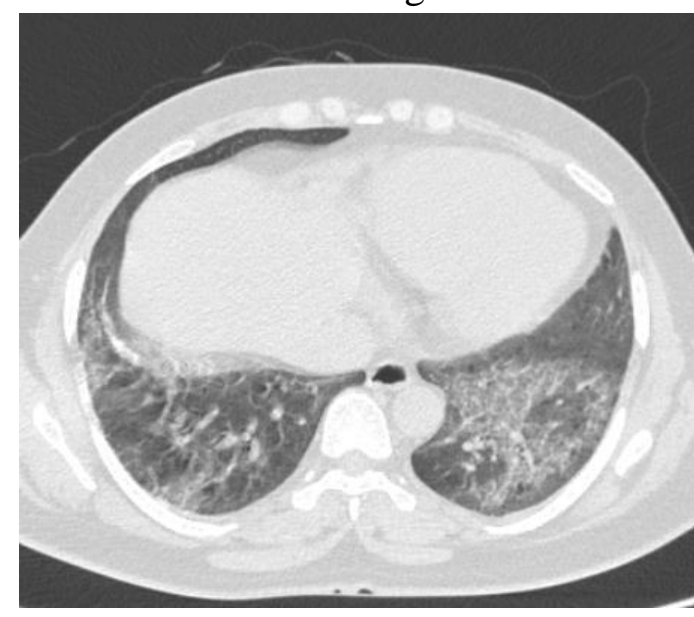

A) CT Image

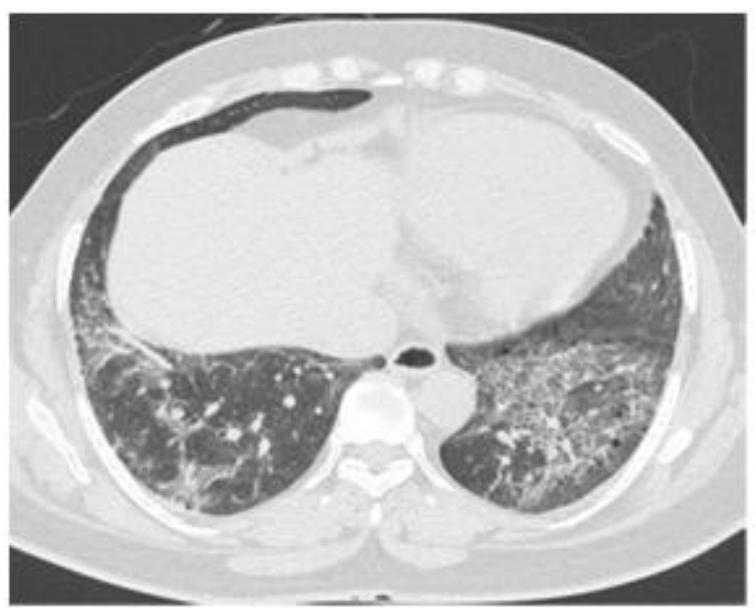

B) HRCT Image

Fig 2: A 38year old female presents with superimposed areas of micro cystic honeycombing noted in posterior basal segments of bilateral lower-lobes-Non-specific interstitial pneumonia type of interstitial lung disease. 


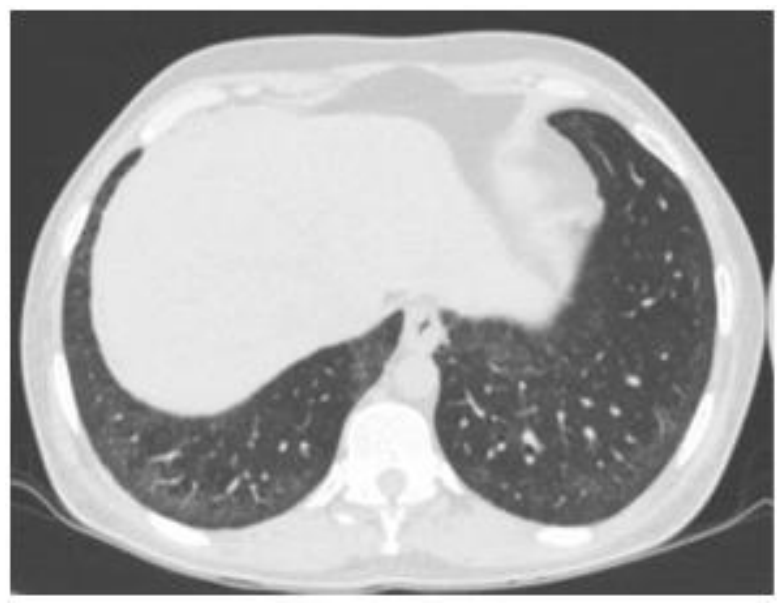

A) CT Image

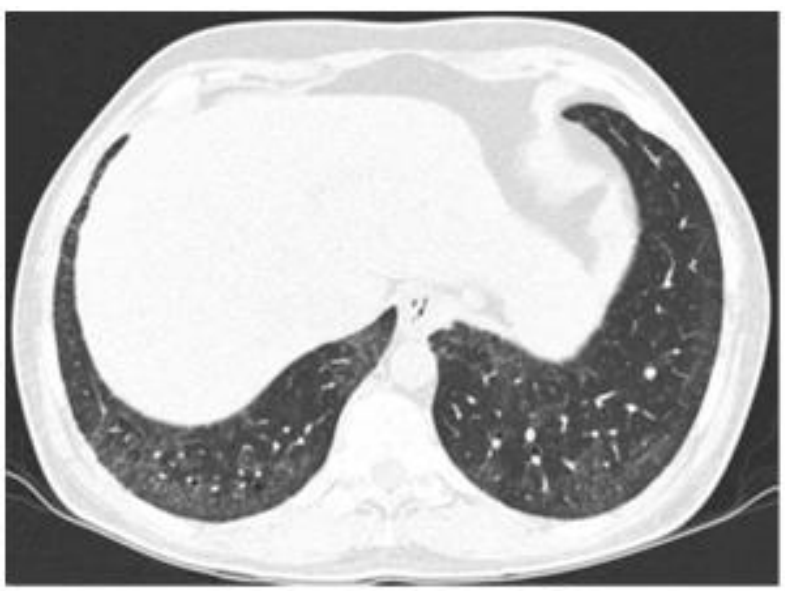

B) HRCT Image

Fig 3: A 42 year male presents with ground glass opacities along with traction bronchiectasis-Nonspecific Interstitial Pneumonia pattern

\section{Conclusion}

On conclusion, HRCT is the most valuable noninvasive modality in diagnosis of interstitial diseases of lung. It even helps to neglect the need of lung biopsies in conjunction with clinical diagnosis. It provides finer details of the lung parenchyma with an excellent spatial resolution whereas Conventional CT fails to demonstrate fine parenchymal details. Thus HRCT helps to make diagnosis with greater confidence.

\section{References}

1. Mayo JR, Webb WR, Gould R, et al. Highresolution CT of the lungs: optimal approach. Radiology 1987; 163:507-510.

2. Murata K, Khan A, Herman PG. Pulmonary parenchymal disease: evaluation with high-resolution CT. Radiology 1989; 170: 629-635.

3. Stein MK, MayoJ, Muller N, Aberle D, Webb WR, Gamsu G. Pulmonary lymphangitic spread of carcinoma: appearance on CT scans. Radiology 1987; 162:371-375.

4. Munk PL, Muller N, Miller RR, Ostrow DN. Pulmonary lymphangiticcarcinomatosis: CT and pathologic findings. Radiology 1988; 166:705-709.

5. Aberle D, Gamsu G, Ray CS, Feurstein IM. Asbestos-related pleural and parenchymal fibrosis: detection with highresolution CT. Radiology 1988; 166:729734.

6. Lynch DA, Gamsu G, Ray CS, Aberle DR. Asbestos-related focal masses: manifestations on conventional and high-resolution CT scans. Radiology 1988; 169:603-607.

7. Murata K, Khan A, Rojas KA, Herman PG. Optimization of computed tomography technique to demonstrate the fine structure of the lung. Invest Radiol 1988; 23:170-175.

8. Gamsu G, Klein J. High-resolution CT of diffuse lung disease. ClinRadiol 1989; 40: 554-556.

9. Staples CA, Gamsu G, Sue Ray C, Webb WR. High-resolution computed tomography and lung function in asbestosexposed workers with normal chest radiographs. Am Rev Respir Dis 1989; 139:1502-1508.

10. Muller NL, Mawson JB, Mathieson JR. Abboud R, Ostrow DN, Champion P. Sarcoi- dosis: correlation of extent of disease at CT with clinical, functional, and radiographic findings. Radiology 1989; 171:613-618.

11. Guidelines for the use of ILO international classification of radiographs of pneumoconiosis. Revised ed. International Labour Office occupational safety and health 
series no. 22 (rev 80). Geneva:

International La- bour Office, 1980.

12. Remy-Jardin M, Beuscart R, Sault MC, Mar- quette CH, Remy J. Subpleuralmicronodules in diffuse mifitrative lung diseases: evaluation with thin-section CT scans. Radiology 1990; 177:133-139.

13. Remy-Jardin M, Degreef JM, Beuscart R, Voisin C, Remy J. Coal worker's pneumoconiosis: CT assessment in exposed workers and correlation with radiographic findings. Radiology 1990; 177:363-371.

14. Zwirewich CV, Terriff B, Muller N. High spatial frequency (bone) algorithm improves quality of standard CT of the tho- rax. AJR 1989; 153:1169-1173. 